\title{
A GENERALIZATION OF THE DISCRETE ISOPERIMETRIC INEQUALITY FOR PIECEWISE SMOOTH CURVES OF CONSTANT GEODESIC CURVATURE
}

\author{
BALÁZs Csikós ${ }^{1}$ (Budapest), Zsolt LÁNGI ${ }^{2}$ (Calgary) and \\ MÁRTON NASZÓDI ${ }^{3}$ (Calgary)
}

Dedicated to Károly Bezdek on his $50^{\text {th }}$ birthday

${ }^{1}$ Dept. of Geometry, Eötvös University, Pázmány Péter Sétány 1/c, 1117 Budapest, Hungary, E-mail: csikos@cs.elte.hu

${ }^{2}$ Dept. of Math. and Stats., University of Calgary, 2500 University Drive NW, Calgary, Ab, T2N 1N4 Canada, E-mail: zlangi@math.ucalgary.ca

${ }^{3}$ Dept. of Math. and Stats., University of Calgary, 2500 University Drive NW, Calgary, Ab, T2N 1N4 Canada, E-mail: nmarton@math.ucalgary.ca

(Received: March 17, 2005; Accepted: November 21, 2005)

\begin{abstract}
The discrete isoperimetric problem is to determine the maximal area polygon with at most $k$ vertices and of a given perimeter. It is a classical fact that the unique optimal polygon on the Euclidean plane is the regular one. The same statement for the hyperbolic plane was proved by Károly Bezdek [1] and on the sphere by László Fejes Tóth [3]. In the present paper we extend the discrete isoperimetric inequality for "polygons" on the three planes of constant curvature bounded by arcs of a given constant geodesic curvature.
\end{abstract}

\section{Introduction}

Throughout this paper, $\mathbf{M}$ denotes any of the three geometries of constant sectional curvature $K \in\{0,-1,1\}$ : the Euclidean plane, $(K=0)$, denoted by $\mathbb{E}^{2}$, the hyperbolic plane, $(K=-1)$, denoted by $\mathbb{H}^{2}$, or the sphere, $(K=1)$, denoted by $\mathbb{S}^{2}$. If $a$ and $b$ are two points of $\mathbf{M}$, which are not antipodal if $\mathbf{M}=\mathbb{S}^{2}$, then $\overline{a b}$ denotes the shortest geodesic segment connecting them.

The discrete isoperimetric problem is to determine the maximal area polygon with at most $k$ vertices and of a given perimeter. It is a classical fact that the unique

Mathematics subject classification number: 51M10, 51M16, 51M25.

Key words and phrases: isoperimetric inequality, Euclidean plane, hyperbolic plane, spherical geometry.

The authors were partially supported by the Hung. Nat. Sci. Found. (OTKA), grant no. T047102, T043556 and T037752. 
optimal polygon for $\mathbf{M}=\mathbb{E}^{2}$ is the regular one. The same statement for $\mathbf{M}=\mathbb{H}^{2}$ was proved by Károly Bezdek [1] and for $\mathbf{M}=\mathbb{S}^{2}$ by László Fejes Tóth [3]. We refer to these results as the (Euclidean, hyperbolic or spherical) discrete isoperimetric inequality. On discrete isoperimetric problems see also [4], [6] and [7]. The following question was asked by Károly Bezdek in personal communication.

Can one extend these results to circle-polygons i.e. "polygons" bounded by circular arcs of a given radius (instead of line segments)?

Convex circle-polygons arise naturally as intersections of finitely many disks of the same radius. This paper answers the question for a slightly more general class of planar figures defined as follows.

Definition. Let $\Gamma \subset \mathbf{M}$ be a simple closed polygon in $\mathbf{M}$ and let $k_{g} \geq 0$ be fixed. If $\mathbf{M}=\mathbb{S}^{2}$, we assume that $\Gamma$ is contained in an open hemisphere. We define the interior and exterior of $\Gamma$ in the usual way for $\mathbf{M}=\mathbb{E}^{2}$ and $\mathbb{H}^{2}$. For $\mathbf{M}=\mathbb{S}^{2}$, the interior of $\Gamma$ is the connected component of $\mathbb{S}^{2} \backslash \Gamma$ having the smaller area. Take the closed curve $P$ obtained by joining consecutive vertices of $\Gamma$ by curves of geodesic curvature $k_{g}$ facing outward (resp. inward), i.e., each curve lies in the half plane bounded by the geodesic connecting the two vertices of $\Gamma$ on the side of the direction of the outer (resp. inner) normal vector of the corresponding side of $\Gamma$. If $k_{g}$ is the geodesic curvature of a circle of radius $r$, then there is an ambiguity in the above definition of $P$ which is fixed by the following two extra conditions: $\Gamma$ is assumed to have sides of length at most $2 r$ and the smooth arcs of $P$ connecting two consecutive vertices are assumed to be shorter than or equal to a semicircle. We call $P$ an outer (resp. inner) $k_{g}$-polygon with the same set of vertices as that of $\Gamma$ with the same cyclic order. We call the arc of $P$ connecting two consecutive vertices $a$ and $b$ a side of $P$ and denote it by $\widehat{a b}$. An ear of $P$ is the region bounded by a side of $\Gamma$ (i.e. $\overline{a b}$ ) and the corresponding side of $P$ (i.e. $\widehat{a b}$ ). We call $\Gamma$ the underlying polygon of $P$. If $\Gamma$ is a regular polygon, then we say that $P$ is regular. The area of an outer $k_{g}$-polygon $P$ is the sum of the area of the interior of the underlying polygon $\Gamma$ and the areas of the ears. This means that if a region is covered more than once by the polygonal domain bounded by $\Gamma$ and the ears, its area is counted with multiplicity. Similarly, the area of an inner $k_{g}$-polygon $P$ is the difference of the area of the interior of $\Gamma$ and the total area of the ears. The perimeter of $P$ is the total length of the sides of $P$. Observe that the perimeter is not necessarily the arc length of the piecewise smooth curve bounding the union (resp. difference) of the interior of $\Gamma$ and the union of the ears. $\mathrm{A}\left(k_{g}, \ell\right)$-polygon is a $k_{g}$-polygon with perimeter $\ell$.

Observe that the discrete isoperimetric problem for the family of $\left(k_{g}, \ell\right)$ polygons with $n$ vertices makes sense only if the parameters $k_{g}, \ell$ and $n$ satisfy the following restrictions, which will be assumed throughout this paper. First, if $k_{g}$ is the geodesic curvature of a circle of radius $r$, then $\ell$ must be less than or equal to $n$ times the length of a semicircle of radius $r$. Second, in the spherical case, if $r n \leq \pi$, then we require also that $\ell$ is less than the perimeter of the $k_{g}$-polygon built around 
the regular $n$-gon inscribed in a great circle. Since the length of a chord of a circle is a concave function of the length of the corresponding arc, the latter assumption implies by Jensen's inequality that the perimeter of the underlying polygon of a $\left(k_{g}, \ell\right)$-polygon cannot exceed the length of a great circle.

Definition. An outer (resp. inner) $\left(k_{g}, \ell\right)$-polygon is optimal if its area is maximal among the areas of outer (resp. inner) $\left(k_{g}, \ell\right)$-polygons having the same number of vertices.

REMARK 1.1. In $\mathbf{M}=\mathbb{E}^{2}$, curves of constant geodesic curvature $k_{g}$ are straight lines when $k_{g}=0$ and circles of radius $r=\frac{1}{k_{g}}$ when $k_{g} \neq 0$. In $\mathbf{M}=\mathbb{S}^{2}$, these curves are circles of radius $r$ with $k_{g}=\cot r$. In $\mathbf{M}=\mathbb{H}^{2}$, they are hyperbolic straight lines for $k_{g}=0$, hypercycles with distance $r$ from a line such that $k_{g}=\tanh r$ for $0<k_{g}<1$, horocycles for $k_{g}=1$ and circles of radius $r$ with $k_{g}=\operatorname{coth} r$ for $1<k_{g}$.

Proposition 1.1. Let $\mathbf{M}$ be $\mathbb{S}^{2}, \mathbb{E}^{2}$ or $\mathbb{H}^{2}$. Let $\ell>0$ and $k_{g} \geq 0$ be given . Then the only optimal inner $\left(k_{g}, \ell\right)$-polygons in $\mathbf{M}$ are the regular ones.

The main result of the paper is the following theorem.

Theorem 1.2. Let $\mathbf{M}$ be $\mathbb{S}^{2}, \mathbb{E}^{2}$ or $\mathbb{H}^{2}$. Let $k_{g} \geq 0, \ell>0$ and $n$ be given with the above restrictions. If $\ell$ is not equal to the perimeter of the circle of geodesic curvature $k_{g}$, then the only optimal outer $\left(k_{g}, \ell\right)$-polygons in $\mathbf{M}$ are the regular ones. If $\ell$ is equal to the perimeter of the circle of geodesic curvature $k_{g}$, then a $\left(k_{g}, \ell\right)$ polygon is optimal if and only if its underlying polygon $\Gamma$ is inscribed in a circle of geodesic curvature $k_{g}$.

We will prove this theorem for $\mathbf{M}=\mathbb{E}^{2}$ in Section 4 by an elementary computational method. The general case will be treated using differential geometric techniques in Section 5.

If $k_{g}$ is the geodesic curvature of a circle of perimeter $\ell$, then according to the classical isoperimetric inequality, a $\left(k_{g}, \ell\right)$-polygon is optimal if and only if its vertices are on a circle of geodesic curvature $k_{g}$. Therefore, in what follows, we assume that $\ell$ is not the perimeter of a circle of geodesic curvature $k_{g}$.

The total area of the ears does not change if the shape of the underlying polygon $\Gamma$ is changed without changing the lengths of the sides. In $\mathbb{E}^{2}$ and $\mathbb{H}^{2}$, a polygon with given side lengths has maximal area if and only if its vertices lie on a curve of constant geodesic curvature. In particular, such a polygon with maximal area must be strictly convex. The same is true on the sphere provided that the perimeter of the polygon is less than that of a great circle. Thus we may and we will assume without loss of generality that $\Gamma$ is strictly convex. Recall that a subset of the sphere is convex if it is the intersection of the sphere and a convex cone with 
vertex at the center of the sphere. A polygonal domain is strictly convex if it is convex and the inner angles of the polygon are all strictly less than $\pi$.

For an overview of results on isoperimetric problems the reader is refered to [2].

\section{Proof of Proposition 1.1}

Proof. Recall that the area of $P$ is the difference of the area of the interior of $\Gamma$ and the total area of the ears of $P$. Using the discrete isoperimetric inequality for polygons in $\mathbf{M}$ it is sufficient to show that if we vary one single vertex remaining in the class of inner $\left(k_{g}, \ell\right)$-polygons, then the total area of the two consecutive ears meeting at that vertex attains its minimum when the ears are congruent. Take a curve $c:[0, \hat{\ell}] \longrightarrow \mathbf{M}$ of constant geodesic curvature $k_{g}$ with endpoints $x_{0}=c(0)$ and $x_{2}=c(\hat{\ell})$ and select a third point $x_{1}$ on the trace of $c$. We show that the total area of the two ears each bounded by a geodesic segment of the form $\overline{x_{i} x_{i+1}}$ and the corresponding arc $\widehat{x_{i} x_{i+1}}$ of $c$ is minimal, when $x_{1}$ is the midpoint of the curve. The area of the domain bounded by $\overline{x_{0} x_{2}}$ and $c$ is fixed, so we want to maximize the area of the triangle $x_{0} x_{1} x_{2}$. In the Euclidean plane it is clearly maximal when $x_{1}$ is in the middle. In $\mathbb{H}^{2}$ and $\mathbb{S}^{2}$, consider the set of points $L(a):=\left\{p \in \mathbf{M}\right.$ : area $\left.\left(x_{0} p x_{2}\right)=a\right\}$ for every $a>0$. This is called the Lexell figure. It is shown in [3] p. 91 that for a given $a$, this set is a pair of equidistant curves (circular arcs on $\mathbb{S}^{2}$, and a pair of hypercycles in $\mathbb{H}^{2}$ ). The Lexell figure is symmetric both about the geodesic segment $\overline{x_{0} x_{2}}$ and its perpendicular bisector. If the area of the triangle $x_{0} x_{1} x_{2}$ is maximal with the constraint $x_{1}$ is on $c$, then the Lexell figure corresponding to that area is tangent to $c$ at $x_{1}$. Since a circle can touch another circle or a hypercycle at most at one point, $x_{1}$ must be in the middle of $c$ in this case by the above symmetry.

This proof also shows that the description of optimal outer $k_{g}$-polygons is not a straightforward corollary of the classical discrete isoperimetric inequality, since as equal sides of $\Gamma$ maximize the area of the region bounded by $\Gamma$, they also minimize the total area of the ears.

\section{Preliminaries}

A standard compactness argument for outer $\left(k_{g}, \ell\right)$-polygons with convex underlying $n$-gons shows the existence of an optimal polygon in this family. We show that if $\ell$ is different from the perimeter of a circle of geodesic curvature $k_{g}$, then an outer $k_{g}$-polygon in $\mathbf{M}$ that is not regular can not be optimal. The main goal of the proof is to show that an optimal outer $k_{g}$-polygon is equilateral, i.e., it has sides of equal length. Once we prove this, the discrete isoperimetric inequality for the underlying polygon shows that the optimal outer $k_{g}$-polygon is also equiangular, i.e., its angles are equal. 
A vertex of a $k_{g}$-polygon $P$ is either a fake vertex or a real one depending on whether the sides of $P$ meeting at that vertex belong to the same smooth curve of constant curvature or not. A fake vertex can freely move along the union of the two sides meeting there without changing the perimeter and the area of $P$. For this reason, if there were an optimal polygon with unequal sides, then moving the fake vertices around we could construct an optimal polygon with two unequal sides meeting at a real vertex. Thus, it is enough to prove that if $P$ is an outer $k_{g}$-polygon with two unequal sides $\widehat{a p}$ and $\widehat{p b}$ meeting at a real vertex $p$, then $P$ is not optimal.

Assume that the side $\widehat{a p}$ is shorter than the side $\widehat{b p}$. If $P$ is optimal, then $p$ must maximize the area $A(p)$ of the figure $F(p)$ bounded by the geodesic segment $\overline{a b}$ and the two sides $\widehat{a p}$ and $\widehat{b p}$ under the constraint that the total length $L(p)$ of $\widehat{a p}$ and $\widehat{b p}$ is fixed. The constraint lets $p$ move along a curve $\tau$, which may have endpoints or may degenerate to a single point. The latter case happens only when both $\widehat{a p}$ and $\widehat{b p}$ are semicircles, but this contradicts the unequality of these sides. $p$ is an endpoint of this curve if and only if the larger side $\widehat{b p}$ is a semicircle as if $\widehat{b p}$ is a semicircle, then $p$ can not move further away from $b$. Even if $p$ is an endpoint of $\tau, p$ can be moved along $\tau$ toward the symmetric position, where the sides $\widehat{a p}$ and $b p$ are equal. To prove that $P$ is not optimal, it suffices to show that the area $A$ increases during this motion. We do this in the next two sections separately for $\mathbb{E}^{2}$ and then for the general case. For an introduction on the differential geometric methods used to prove the general case see, for example, [5].

We assume that $k_{g}>0$, as the case $k_{g}=0$ is the discrete isoperimetric inequality for polygons.

Definition. Let $q \in \mathbf{M}$ be an arbitrary point and $k_{g} \geq 0$ be given. We define the $k_{g}$-arc-length function $f_{q}$ for points of $\mathbf{M}$ that can be connected to $q$ by a curve of constant geodesic curvature $k_{g}$ as the arc-length of a shortest such curve.

\section{Proof of Theorem 1.2 in the Euclidean plane}

Proof of Theorem 1.2 FOr $\mathbf{M}=\mathbb{E}^{2}$. In the Euclidean plane all curves of non-zero constant geodesic curvature are circular arcs. Without loss of generality we assume that $k_{g}=1$, i. e. the radius of the circles is 1 . Introduce the notations $\alpha:=f_{a}(p), \beta:=f_{b}(p)$, and $d:=\frac{\operatorname{dist}(a, b)}{2}$. As it was assumed in Section 3, we have $\alpha<\beta$. Let $\rho:=\alpha+\beta$ be fixed, then $\beta=\rho-\alpha$ becomes a function of $\alpha$.

We consider the area $A$ of the figure $F(p)$ as a function of $\alpha$ with parameters $d$ and $\rho$. We show that the derivative of $A$ with respect to $\alpha$ is positive.

Using Heron's formula for the area of the triangle we have

$$
\begin{gathered}
A(\alpha)=\frac{\alpha-\sin \alpha}{2}+\frac{\beta-\sin \beta}{2}+T \\
=\frac{\alpha+\beta}{2}-\frac{\sin \alpha+\sin \beta}{2}+\sqrt{2 d^{2}-d^{4}-\frac{(\cos \alpha-\cos \beta)^{2}}{4}-(\cos \alpha+\cos \beta) d^{2}},
\end{gathered}
$$


where $T$ denotes the area of the Euclidean triangle $a p b$. The derivative of $A$ is

$$
A^{\prime}(\alpha)=-\frac{\cos \alpha-\cos \beta}{2}+\frac{1}{2 T}\left[\frac{(\cos \alpha-\cos \beta)(\sin \alpha+\sin \beta)}{2}+(\sin \alpha-\sin \beta) d^{2}\right] \text {. }
$$

By trigonometric identities we get that

$$
A^{\prime}(\alpha)=-\frac{\cos \alpha-\cos \beta}{2 T}\left[T-\frac{\sin \alpha+\sin \beta}{2}+d^{2} \cot \frac{\rho}{2}\right] .
$$

Set $B:=\frac{\sin \alpha+\sin \beta}{2}-d^{2} \cot \frac{\rho}{2}$. As $\cos \alpha>\cos \beta$ and $T>0$, in order to show that $A^{\prime}(\alpha)>0$ we need to show that $T-B<0$. Observe that

$$
T^{2}-B^{2}=\frac{-2\left(d^{2}-\sin ^{2} \frac{\rho}{2}\right)^{2}}{1-\cos \rho} .
$$

This expression is nonpositive, and it is zero only when $d=\sin \frac{\rho}{2}$, i.e. when the points $a, p$ and $b$ are on a unit circular arc, which contradicts our assumptions.

As $T$ is clearly positive, to prove our statement it is enough to show that $B>0$. We regard $B$ as a function of $d$ with parameters $\alpha$ and $\beta$. Obviously, $B(d)$ is continuous on $\mathbb{R}$, and $B(0)>0$. As $T$ is positive, and $B^{2} \geq T^{2}, B$ does not change sign on the interval where $T$ is defined, i.e. on the interval $\left(0, d_{\max }\right)$, where $d_{\max }=\sin \frac{\alpha}{2}+\sin \frac{\beta}{2}$. As a result, we have $B(d)>0$ for any $d \in\left(0, d_{\max }\right)$. This finishes the proof of Theorem 1.2 for the Euclidean plane.

\section{Proof of Theorem 1.2 in the general case}

The following arguments hold in all the three geometries.

Notation. Let $\mathbf{t}_{a}, \mathbf{t}_{b} \in T_{p} \mathbf{M}$ be the unit tangent vectors of the oriented sides $\widehat{p a}$ and $\widehat{p b}$ (directed from $p$ to $a$ and from $p$ to $b$, respectively). Let $\mathbf{t}_{e}:=\frac{\mathbf{t}_{b}-\mathbf{t}_{a}}{\left\|\mathbf{t}_{b}-\mathbf{t}_{a}\right\|}$ be a unit vector in $T_{p} \mathbf{M}$ in the direction of the external angular bisector of $\mathbf{t}_{a}$ and $\mathbf{t}_{b}$.

If $F$ is locally convex at $p$, then let $\mathbf{t}_{i}:=\frac{\mathbf{t}_{a}+\mathbf{t}_{b}}{\left\|\mathbf{t}_{a}+\mathbf{t}_{b}\right\|}$. If $F$ is not locally convex at $p$, then let $\mathbf{t}_{i}:=-\frac{\mathbf{t}_{a}+\mathbf{t}_{b}}{\left\|\mathbf{t}_{a}+\mathbf{t}_{b}\right\|}$. I.e., $\mathbf{t}_{i}$ is a unit vector in $T_{p} \mathbf{M}$ in the direction of the internal angular bisector of $\mathbf{t}_{a}$ and $\mathbf{t}_{b}$.

Let $0<\gamma<\pi$ be the angle between $\mathbf{t}_{a}$ and $\mathbf{t}_{i}$. Since the two sides $\widehat{a p}$ and $\widehat{b p}$ are not parts of the same curve of constant geodesic curvature $k_{g}$, we have $\gamma \neq \frac{\pi}{2}$.

Let $0<\sigma_{a} \leq \frac{\pi}{2}$ (and $0<\sigma_{b} \leq \frac{\pi}{2}$ ) denote the angle between the geodesic segment $\overline{p a}$ (resp. $\overline{p b})$ and the curve $\widehat{p a}$ (resp. $\widehat{p b})$. (See Figure 1.)

ClaIm 5.1. Let $\mathbf{M}$ be $\mathbb{E}^{2}, \mathbb{H}^{2}$ or $\mathbb{S}^{2}$. Using the above notations suppose that the arc $\widehat{p a}$ is not a semicircle. Let $\mathbf{t} \in T_{p} \mathbf{M}$ be a unit vector such that the oriented angle between $\mathbf{t}_{a}$ and $\mathbf{t}$ is $\phi$, where the orientation is given by the ordered basis $\left(\mathbf{t}_{a}, \mathbf{t}_{i}\right)$. Then the derivative of the $k_{g}$-arc-length function $f_{a}$ in the direction of $\mathbf{t}$ is

$$
\mathbf{t}\left(f_{a}\right)=-\cos \phi-\sin \phi \tan \sigma_{a} .
$$


Proof. Let $\mathbf{u}, \mathbf{v} \in T_{p} \mathbf{M}$ be an orthonormal basis in $T_{p} \mathbf{M}$ such that $\mathbf{v}$ is tangent to the geodesic $\overline{p a}$ and points toward $a$.

Set $d_{a}: \mathbf{M} \longrightarrow \mathbb{R}: x \mapsto \operatorname{dist}(a, x)$. Clearly, $\mathbf{u}\left(d_{a}\right)=0$, hence $\mathbf{u}\left(f_{a}\right)=0$. On the other hand, let $c:(-\varepsilon, \varepsilon) \longrightarrow \mathbf{M}$ be the arc length parametrization of the curve of constant geodesic curvature $k_{g}$ such that $c(0)=p$ and $c([0, \varepsilon]) \subset \widehat{p a}$. The vector $\mathbf{t}_{a}$ can be represented as the differentiation along the curve $c$ at 0 , thus

$$
\mathbf{t}_{a}\left(f_{a}\right)=\left.\frac{\mathrm{d}}{\mathrm{d} t} f_{a}(c(t))\right|_{t=0}=-1 \quad \text { and } \quad \mathbf{u}\left(f_{a}\right)=0 .
$$

Since $\widehat{p a}$ is not a semicircle, $\mathbf{u}$ and $\mathbf{t}_{a}$ are not parallel and we can decompose $\mathbf{t}$ as a linear combination $\mathbf{t}=\mu \mathbf{t}_{a}+\lambda \mathbf{u}$ of them. Equation (1) and this decomposition yields

$$
\mathbf{t}\left(f_{a}\right)=\mu \mathbf{t}_{a}\left(f_{a}\right)=-\mu=-\frac{\langle\mathbf{t}, \mathbf{v}\rangle}{\left\langle\mathbf{t}_{a}, \mathbf{v}\right\rangle}=-\frac{\cos \left(\phi-\sigma_{a}\right)}{\cos \sigma_{a}} .
$$

By the addition formula for the cosine function we finish the proof of the claim.

Lemma 5.1. We use the above notations. Suppose that the inner angle of $\Gamma$ at $p$ is strictly convex and that neither $\widehat{p a}$ nor $\widehat{p b}$ is a semicircle i.e. $\sigma_{a} \neq \frac{\pi}{2}$ and $\sigma_{b} \neq \frac{\pi}{2}$. Then

$$
\begin{gathered}
L_{e}:=\mathbf{t}_{e}(L)=\cos \gamma\left(\tan \sigma_{b}-\tan \sigma_{a}\right) . \\
L_{i}:=\mathbf{t}_{i}(L)=-2 \cos \gamma-\sin \gamma\left(\tan \sigma_{a}+\tan \sigma_{b}\right) .
\end{gathered}
$$

Moreover, if we set $\mathbf{x}:=-L_{i} \mathbf{t}_{e}+L_{e} \mathbf{t}_{i}$, then

$$
\mathbf{x}(L)=0 \quad \text { and } \quad \mathbf{x}\left(f_{a}\right)>0 .
$$

Proof. Equations (2) and (3) follow from Claim 5.1 using that the oriented angle between $\mathbf{t}_{a}$ and $\mathbf{t}_{i}$ is $\gamma$ as well as between $\mathbf{t}_{b}$ and $\mathbf{t}_{i}$, while the oriented angle between $\mathbf{t}_{a}$ and $\mathbf{t}_{e}$ is $\gamma+\frac{\pi}{2}$ and between $\mathbf{t}_{b}$ and $\mathbf{t}_{i}$ is $\gamma-\frac{\pi}{2}$. The first formula in (4) is obvious.

By substituting Equations (2) and (3) in the definition of $\mathbf{x}$ and using Claim 5.1, after simplifications we have

$$
\mathbf{x}\left(f_{a}\right)=\frac{\sin \left(2 \gamma-\sigma_{a}-\sigma_{b}\right)}{\cos \sigma_{a} \cos \sigma_{b}}>0 .
$$

The inequality holds, since $2 \gamma-\sigma_{a}-\sigma_{b}$ is the angle of the triangle $a p b$ at $p$ so, $0<2 \gamma-\sigma_{a}-\sigma_{b}<\pi$ and $0<\sigma_{a}, \sigma_{b}<\frac{\pi}{2}$.

Recall from Section 3 that we prove Theorem 1.2 by a local argument. We are going to show that moving $p$ along a level curve $\tau$ of $f_{a}+f_{b}$ in the direction in which the difference $\left|f_{b}-f_{a}\right|$ decreases the area $A$ is increasing. Because of continuity of $A$, it is enough to prove monotonicity of $A$ on the relative interior of the curve $\tau$. According to Lemma 5.1, if $p$ is not an endpoint of $\tau$, then the vector $\mathbf{x}=-L_{i} \mathbf{t}_{e}+L_{e} \mathbf{t}_{i}$ is tangent to $\tau$ and points toward the symmetric position, so to 


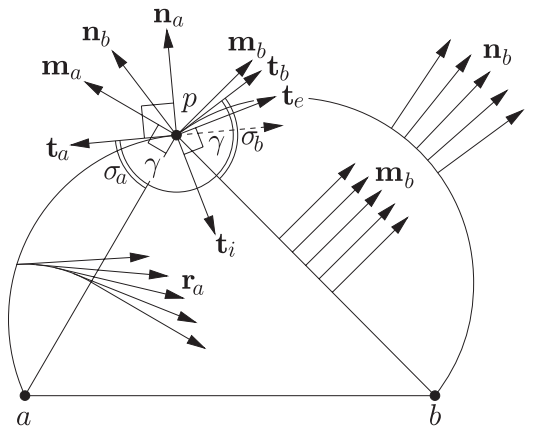

FiguRe 1

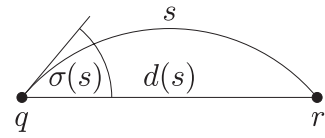

FiguRe 2

finish the proof, all we have to check is that $\mathbf{x}(A)>0$. For this purpose, we shall compute the differential of $A$ explicitly.

The following claim collects some relations between the geometric data of an ear. The proof of these relations is straightforward from known identities of hyperbolic, Euclidean and spherical trigonometry, so we omit it.

In the following claim we collect three formulae and omit the proof.

Claim 5.2. Let $\tau \subset \mathbf{M}$ be a curve of constant geodesic curvature $k_{g}$ of arc length $s$ with endpoints $q$ and $r, K$ the sectional curvature of $\mathbf{M}$. Suppose that if $k_{g}^{2}+K>0$, then $\tau$ is shorter than a semicircle, i.e., $s<\frac{\pi}{\sqrt{k_{g}^{2}+K}}$. Let $\sigma(s)$ denote the angle between $\tau$ and the geodesic segment $\overline{q r}$ and let $d(s):=\operatorname{dist}(q, r)$, see Figure 2. Then,

$$
\begin{gathered}
\tan \sigma(s)=\frac{k_{g}}{\sqrt{k_{g}^{2}+K}} \tan \frac{s \sqrt{k_{g}^{2}+K}}{2}, \quad \sin \frac{\sqrt{K} d(s)}{2}=\sqrt{\frac{K}{k_{g}^{2}+K}} \sin \frac{s \sqrt{k_{g}^{2}+K}}{2}, \\
\frac{\sin (\sqrt{K} d(s))}{\sqrt{K}} \cos \sigma(s)=\frac{\sin \left(s \sqrt{k_{g}^{2}+K}\right)}{\sqrt{k_{g}^{2}+K}} .
\end{gathered}
$$

These formulae make sense as the functions $\sin$ and $z \mapsto \frac{\sin z}{z}$ are regarded as complex holomorphic functions defined on the whole complex plane. Similarly, tan and $z \mapsto \frac{\tan z}{z}$ are regarded as meromorphic functions having poles only at odd multiples of $\pi / 2$.

LEMma 5.2. With the above notations, the derivatives of the area $A(p)$ (defined in Section 3) in the directions $\mathbf{t}_{e}$ and $\mathbf{t}_{i}$, respectively, are

$$
A_{e}:=\mathbf{t}_{e}(A)=\frac{\cos \gamma}{\sqrt{k_{g}^{2}+K}}\left(\tan \frac{f_{b}(p) \sqrt{k_{g}^{2}+K}}{2}-\tan \frac{f_{a}(p) \sqrt{k_{g}^{2}+K}}{2}\right),
$$




$$
A_{i}:=\mathbf{t}_{i}(A)=-\frac{\sin \gamma}{\sqrt{k_{g}^{2}+K}}\left(\tan \frac{f_{b}(p) \sqrt{k_{g}^{2}+K}}{2}+\tan \frac{f_{a}(p) \sqrt{k_{g}^{2}+K}}{2}\right) .
$$

Proof. For $\mathbf{w} \in T_{p} \mathbf{M}$, we compute the derivative $\mathbf{w}(A)$ as follows.

Choose a curve $\eta:(-\epsilon, \epsilon) \rightarrow \mathbf{M}$ describing a motion of $\eta(0)=p$ with initial speed $\eta^{\prime}(0)=\mathbf{w}$. Let $*$ stand for $a$ or $b$. Take the unit speed parametrization $\zeta_{*}: \mathbb{R} \rightarrow \mathbf{M}$ of the curve of constant geodesic curvature $k_{g}$ containing the arc $\widehat{* p}$ such that $\zeta(0)=*$ and $\zeta\left(f_{*}(p)\right)=p$. Denote by $R_{*}(q, \theta)$ the rotation of $q \in \mathbf{M}$ about $*$ with angle $\theta$ with respect to a fixed orientation of $\mathbf{M}$. Rotations about $*$ form a one parameter group of isometries generated by the Killing field $\mathbf{r}_{*}$, where $\mathbf{r}_{*}(q)=\partial_{\theta} R_{*}(q, 0)$ (see Figure 1). There is a smooth function $\theta_{*}:(-\epsilon, \epsilon) \rightarrow \mathbb{R}$ such that the map $[0,1] \rightarrow \mathbf{M}, u \mapsto R_{*}\left(\zeta_{*}\left(u f_{*}(\eta(t))\right), \theta_{*}(t)\right)$ is a parametrization of the side $\widehat{* \eta(t)}$ of the domain $F(\eta(t))$. The initial speed vector field of this variation of the side $\widehat{* p}$ is the vector field $\mathbf{v}_{*}$ along the curve $\widehat{* p}$ the value of which at the point $q=\zeta_{*}\left(u f_{*}(p)\right)$ is computed by the formula

$$
\mathbf{v}_{*}(q)=\left.\frac{\partial}{\partial t} R_{*}\left(\zeta_{*}\left(u f_{*}(\eta(t))\right), \theta_{*}(t)\right)\right|_{t=0}=u \mathbf{w}\left(f_{*}\right) \zeta_{*}^{\prime}\left(u f_{*}(p)\right)+\theta_{*}^{\prime}(0) \mathbf{r}_{*}(q) .
$$

The speed vector $\mathbf{v}_{*}(p)$ coincides with the speed vector $\mathbf{w}$ of $p$, i.e.,

$$
\mathbf{w}=-\mathbf{w}\left(f_{*}\right) \mathbf{t}_{*}+\theta_{*}^{\prime}(0) \mathbf{r}_{*}(p),
$$

Let $\mathbf{n}_{*}$ be the outer unit vector field along and orthogonal to the side $\widehat{* p}$. The derivative of the function $A$ with respect to $\mathbf{w}$ can be expressed as

$$
\mathbf{w}(A)=\int_{\widehat{a p}}\left\langle\mathbf{n}_{a}, \mathbf{v}_{a}\right\rangle d s+\int_{\widehat{b p}}\left\langle\mathbf{n}_{b}, \mathbf{v}_{b}\right\rangle d s=\sum_{* \in\{a, b\}} \theta_{*}^{\prime}(0) \int_{\widehat{* p}}\left\langle\mathbf{n}_{*}, \mathbf{r}_{*}\right\rangle d s .
$$

Since $\mathbf{r}_{*}$ is a Killing field and therefore it is divergence free, denoting by $\mathbf{m}_{*}$ the outer unit normal of the polygon $\Gamma$ along the side $\overline{* p}$, we have

$$
\int_{\widehat{* p}}\left\langle\mathbf{n}_{*}, \mathbf{r}_{*}\right\rangle d s=\int_{\overline{* p}}\left\langle\mathbf{m}_{*}, \mathbf{r}_{*}\right\rangle d s .
$$

The latter integral can be computed easily using the explicit form $\mathbf{r}_{*}= \pm \frac{\sin \left(\sqrt{K} d_{*}\right)}{\sqrt{K}} \mathbf{m}_{*}$ of the vector field $\mathbf{r}_{*}$ along the segment $\overline{* p}$, where $d_{*}(q)$ is the geodesic distance of $q \in \mathbf{M}$ from the point $*$ :

$$
\begin{aligned}
\int_{\overline{* p}}\left\langle\mathbf{m}_{*}, \mathbf{r}_{*}\right\rangle d s & = \pm \frac{1-\cos \left(\sqrt{K} d_{*}(p)\right)}{K}=\left\langle\mathbf{r}_{*}(p), \mathbf{m}_{*}(p)\right\rangle \frac{1-\cos \left(\sqrt{K} d_{*}(p)\right)}{\sqrt{K} \sin \left(\sqrt{K} d_{*}(p)\right)} \\
& =\frac{\left\langle\mathbf{r}_{*}(p), \mathbf{m}_{*}(p)\right\rangle}{\sqrt{K}} \tan \left(\frac{\sqrt{K} d_{*}(p)}{2}\right) .
\end{aligned}
$$



of $A$

From (5), (6) and (7) we obtain the following general formula for the derivative

$$
\begin{aligned}
\mathbf{w}(A) & =\sum_{* \in\{a, b\}} \theta_{*}^{\prime}(0) \frac{\left\langle\mathbf{r}_{*}(p), \mathbf{m}_{*}(p)\right\rangle}{\sqrt{K}} \tan \left(\frac{\sqrt{K} d_{*}(p)}{2}\right) \\
& =\sum_{* \in\{a, b\}} \frac{\left(\left\langle\mathbf{w}, \mathbf{m}_{*}(p)\right\rangle+\mathbf{w}\left(f_{*}\right)\left\langle\mathbf{t}_{*}, \mathbf{m}_{*}(p)\right\rangle\right)}{\sqrt{K}} \tan \left(\frac{\sqrt{K} d_{*}(p)}{2}\right) .
\end{aligned}
$$

In the special cases, when $\mathbf{w}=\mathbf{t}_{i}$ or $\mathbf{w}=\mathbf{t}_{e}$, the angles between the unit vectors $\mathbf{w}, \mathbf{m}_{*}(p)$ and $\mathbf{t}_{*}$ are known explicitly and an explicit expression for $\mathbf{w}\left(f_{*}\right)$ is also given in Claim 5.1. Substituting these explicit values into (8) we obtain

$$
\begin{aligned}
\mathbf{t}_{i}(A) & =\sum_{* \in\{a, b\}}\left(\cos \left(\frac{\pi}{2}+\gamma-\sigma_{*}\right)-\frac{\cos \left(\gamma-\sigma_{*}\right)}{\cos \sigma_{*}} \cos \left(\frac{\pi}{2}-\sigma_{*}\right)\right) \frac{\tan \left(\frac{\sqrt{K} d_{*}(p)}{2}\right)}{\sqrt{K}} \\
& =-\sum_{* \in\{a, b\}} \frac{\sin \gamma}{\sqrt{K} \cos \sigma_{*}} \tan \left(\frac{\sqrt{K} d_{*}(p)}{2}\right)
\end{aligned}
$$

and similarly,

$$
\mathbf{t}_{e}(A)=-\frac{\cos \gamma}{\sqrt{K} \cos \sigma_{a}} \tan \left(\frac{\sqrt{K} d_{a}(p)}{2}\right)+\frac{\cos \gamma}{\sqrt{K} \cos \sigma_{b}} \tan \left(\frac{\sqrt{K} d_{b}(p)}{2}\right)
$$

The equivalence of the last two equations and the equations we wanted to show follows easily from the formulae of Claim 5.2.

Proof of TheOREm 1.2. We have already explained that all we have to show is that for $\mathbf{x}=-L_{i} \mathbf{t}_{e}+L_{e} \mathbf{t}_{i}$, we have $\mathbf{x}(A)=-L_{i} A_{e}+L_{e} A_{i}>0$ provided that $f_{b}(p)>f_{a}(p)$. Computing $\mathbf{x}(A)$ explicitly using Lemmas 5.1 and 5.2, then simplifying the result with the help of the first equation of Claim 5.2 we end up with

$$
\mathbf{x}(A)=\frac{2 \cos ^{2} \gamma}{\sqrt{k_{g}^{2}+K}}\left(\tan \frac{f_{b}(p) \sqrt{k_{g}^{2}+K}}{2}-\tan \frac{f_{a}(p) \sqrt{k_{g}^{2}+K}}{2}\right) .
$$

As the right hand side is obviously positive when $f_{b}(p)>f_{a}(p)$, this finishes the proof of the theorem. 


\section{Concluding Remarks}

When $k_{g}$ is the geodesic curvature of a circle, then we can consider the discrete isoperimetric problem for outer $k_{g}$-polygons for which all the sides are circular arcs longer than a semicircle. Obviously, the problem for "big eared" outer $k_{g}$-polygons can be reduced to Proposition 1.1 since a big eared $k_{g}$-polygon is optimal if and only if the small eared inner $k_{g}$-polygon built around the same underlying polygon is optimal.

The authors are grateful for Prof. Károly Bezdek for raising the question and for his continuous support in the research.

\section{References}

[1] K. BEzDEK, Ein elementarer Beweis für die isoperimetrische Ungleichung in der Euklidischen und hyperbolischen Ebene, Annales Universitatis Scientiarum Budapestiensis de Rolando Eötvös Nominatae, Sectio Mathematica 27 (1985), 107112.

[2] Yu. D. Burago and V. A. Zalgaller, Geometric Inequalities, (transl. from the Russian by A. B. Sossinsky), Grundlehren der Mathematischen Wissenschaften, 285, Berlin, etc., Springer-Verlag, 1988.

[3] L. Fejes Tóth, Regular figures, Pergamon Press, 1964.

[4] H. S. M. Coxeter, Regular polytopes, 3rd edn, Dover Publications, Inc., New York, 1973.

[5] M. Do CARmo, Differential geometry of curves and surfaces, Prentice Hall, Englewood Cliffs, 1976.

[6] B. Datta, A discrete isoperimetric problem, Geom. Dedicata 64 (1997), no. 1, $55-68$.

[7] Hsu-Tung Ku, Mei-Chin Ku and Xin-Min Zhang, Isoperimetric inequalities on surfaces of constant curvature, Canad. J. Math. 49 (1997), no. 6, 1162-1187. 